\title{
3D Reconstruction and Rendering Models in Urban Architectural Design Using Kalman Filter Correction Algorithm
}

\author{
Kaihong Wang $(\mathbb{D}$ and Xuelun Guan \\ Guangdong University of Foreign Studies, College of Arts, Guangzhou 510420, China \\ Correspondence should be addressed to Kaihong Wang; wangkh@gdufs.edu.cn
}

Received 17 January 2022; Revised 13 February 2022; Accepted 14 February 2022; Published 3 March 2022

Academic Editor: Xin Ning

Copyright (c) 2022 Kaihong Wang and Xuelun Guan. This is an open access article distributed under the Creative Commons Attribution License, which permits unrestricted use, distribution, and reproduction in any medium, provided the original work is properly cited.

\begin{abstract}
In a virtual 3D city scene, a 3D building model is a must-have element. A 3D reconstruction and rendering algorithm is described in this paper. Model geometry and texture data are simplified separately using LOD model technology. Half-folded mesh algorithm is used to simplify geometric data, while wavelet algorithm is used to compress texture data. Both methods reduce the amount of data that must be stored as well as the amount of data that must be transmitted over the network. To improve the response time of the original algorithm, a Kalman filter correction algorithm is used to optimize the 3D reconstruction beam adjustment algorithm. In this paper, the experimental scheme is used to assess the method. Experiments show that the algorithm reduces the number of primitives drawn by the system while preserving the important geometric features of the scene's building model. It can also effectively reduce the workload of drawing 3D scenes, improve computer productivity, and reduce computer hardware requirements. This technique is well suited to rendering large-scale $3 \mathrm{D}$ urban scenes.
\end{abstract}

\section{Introduction}

With the development of social economy and cities in China and the need of new urbanization, how to ensure the coordinated development of economy, culture, science and technology, politics, etc. of cities and follow the development concept of being people-oriented put forward new requirements and challenges for city managers [1]. The city is a symbol of the digital earth, and it is also an indispensable part in the construction of the digital earth. 3D model visualization of urban landscape is one of the important technologies of digital city, which is widely used in many fields such as architecture, transportation, and urban planning [2]. The increasing complexity and data density of 3D digital models bring great pressure to computer storage, display, transmission, and rendering [3]. Traditional urban planning is to build a two-dimensional urban spatial information management system with the support of Geographic Information System (GIS) technology, which can meet the basic needs of city managers such as information query and result display, and the needs of urban development simulation and spatial analysis cannot be well solved due to technical prob- lems. With the development of 3D technology, the transformation from $2 \mathrm{D}$ to $3 \mathrm{D}$ is an inevitable trend [4]. Traditional $3 \mathrm{D}$ reconstruction methods of buildings have defects in efficiency, accuracy, cost, scale, technical threshold, etc. in the face of urban buildings with large spatial scale, large amount of data, and fast updating pace. How to quantitatively optimize the detail level of the model according to the different complexity of the model, adaptively retain important features, and reduce unnecessary redundancy, so as to conform to the hierarchy of people's cognitive process, is an important technical content of Web3D display.

At present, the society has entered the era of large-scale digitalization, and all subsystems in the city where we live are gradually turning into informationization. Virtual communities, virtual businesses, online virtual schools, virtual banks, and so on have emerged one after another [5]. Traditional urban planning is mainly represented by handdrawing, architectural miniature model, architectural renderings, and 3D animation [6]. With the rapid development of digital city and the continuous expansion of the application field of urban 3D spatial model, the demand for 3D model of urban buildings is growing. Complex scenes in 
3D cities frequently necessitate large amounts of multisource and heterogeneous spatial data, and a 3D building model is an essential component [7]. It is necessary to create different levels of detail models for the original high-resolution mesh model in order to get architectural models suitable for various scenes. The rationality, economy, ecological function, aesthetics, and art of urban architecture and landscape planning should all be considered in urban planning [8]. Urban planning has obvious continuity and advancement characteristics, and the rapid pace of urban informatization raises the stakes for urban planning, construction, and management. Simulation technology has evolved into a critical component of urban planning. The 3D city model has been widely used as a key spatial data infrastructure [9]. The need for large-scale 3D scene rendering is growing in popularity. More urgent application requirements exist, particularly in the fields of urban planning, road traffic, property management, cadastral management, water conservation, and electric power. 3D building models have a larger amount of data and more complex geometric features than other types of data in urban scenes [10]. The first problem to be solved right now is how to display so many $3 \mathrm{D}$ models quickly and smoothly. This paper examines urban architectural design and delves into the key $3 \mathrm{D}$ rendering technologies.

It is the core space of modern human life in cities. Under the background of urban informatization, local governments are aimed at digital cities and smart cities and carrying out urban digital construction [11]. With the development of digital earth, digital city, and digital community, massive 3D model visualization technology has become an important direction of current development [12]. The analysis of 3D reconstruction in the field of computer vision is the research of feature inspection. Conventional features include feature points, feature lines, and feature areas [13]. With the development of computer technology, on the one hand, the ability of map expression and spatial confidence transmission is greatly improved; on the other hand, it is conducive to the understanding of spatial information and provides more secure decision-making services. Among them, there are many examples to study the characteristics of using points. At present, domestic governments and functional departments at all levels realize that urban informatization is the trend of urban construction and planning, and how to strengthen urban informatization construction has been put on the agenda and is in full swing [14]. Urban informatization planning is also in front of urban managers, and it is the trend of urban construction to build 3D urban planning decision-making system. Aiming at the problem that existing 3D reconstruction and rendering algorithms cannot meet the needs of 3D reconstruction of urban landscape, this paper proposes a 3D reconstruction algorithm based on Kalman filter correction and a terrain rendering algorithm based on level of detail (LOD). Based on the analysis of urban 3D building model structure, a new 3D model data structure is defined according to the requirements of system functions in the future. The simulation results show that the algorithm proposed in this paper works well in the application of 3D reconstruction of urban landscape. It has certain practicability.

\section{Related Work}

In the field of camera calibration, literature [15] proposed a flat template calibration method. In terms of the 3D reconstruction algorithm, literature [16] demonstrates how to create a texture mapping 3D model of a building from an image sequence. By dividing the earth's surface into grids, literature [17] transforms global 3D building data management into $3 \mathrm{D}$ building data management in a single grid. The database organization and management of $3 \mathrm{D}$ buildings in a single grid are completed, the database table is created, and temporal data management is considered all at once. While the database's basic functions are implemented, features such as historical data archiving are added. Literature [18] uses a single mobile camera to create a three-dimensional scene of historical scenic spots, demonstrating that in-depth information on the scene's surface can be obtained with a quick and inexpensive device. The Canny operator has a certain display significance in the function and efficiency of the motion recovery structure, according to literature [19]. By decomposing the structure of the building voxel, literature [20] established the relationship description of the 3D model objects of a single building. Use the least square method to construct a 3D model of a single building using typical geometric constraint conditions, and obtain preliminary research results. Automatically detect buildings to extract surface information from urban scenes. Literature [21] proposed a scheduling strategy for 3D building data, used grids and $\mathrm{KD}$ trees to index the model, used multithreading technology to achieve parallel processing of 3D model display, and used caching technology to reduce system-database interaction. In massive $3 \mathrm{D}$ visualization system for buildings, literature [22] studied the 3D automatic reconstruction of regular houses based on multicue recognition and discussed the role of uncertainty reasoning theory in recognition verification. Literature [23] uses the method of geometric structural element analysis to extract various basic structural elements that form a rectangle in the graph and then merge the basic structural elements into a rectangular structure and automatically extract the rectangular buildings in the aerial city image. Literature [24] uses the data as prior knowledge to give the location and overall outline of the building, then uses the building material center in the image as the initial seed point for region segmentation using fuzzy sets, and finally uses adaptive threshold segmentation for the obtained results. In morphological method for trimming, literature [25] used unsupervised clustering of high-resolution satellite panchromatic images to confirm the existence of the building by looking at the shadow area of the building with the lowest gray value in the result and then performed edge extraction and transformation processing on the candidate target to obtain architectural goals. Based on the data characteristics and attribute information of the 3D building model, literature [26] analyzes the data hierarchy of the 3D building model and designed the data structure for the 3D building model, as well as organized and managed the algorithm's 3D building LOD model. A shadow-assisted building extraction method was proposed in the literature [27]. This method extracts buildings and shadow objects from high- 
resolution images, then separates the interconnected building objects based on the spatial relationship between the buildings and their shadows, confirming the missing buildings and improving building extraction accuracy. For complex and irregular buildings, literature [28] first extracts feature points and judges their attributes, then performs conditional adjustment on feature points at right angles, and optimizes the location of feature points. Arc fitting is used to obtain a smooth contour line that conforms to the actual situation for the boundary segmentation between the feature points at the arc. This method is capable of extracting building contours with arcs.

The article researches and analyzes the key technologies of urban building design and rendering. Explore a low-cost, low-threshold, and high-efficiency 3D reconstruction rendering solution suitable for urban buildings to meet the urgent needs of digital cities and related fields. This paper optimizes the LOD terrain rendering algorithm; uses the node evaluation function to determine whether the node needs to be split, so as to achieve different levels of detail; and uses the method of adding edges and deleting edges to eliminate cracks in the terrain. The grid storage of 3D building model data is realized by dividing the center coordinates of building model with equal latitude and longitude difference grid, and the data with different scopes of action are stored in layers and blocks by pyramid structure, so as to improve the index efficiency of data and the speed of network response. Simulation experiments are carried out to verify the feasibility of the whole solution from the aspects of accuracy, efficiency, and timeliness.

\section{Methodology}

3.1. 3D Model Data Foundation. The 3D model is the 3D urban planning auxiliary decision-making system's main filling, display, and analysis content. Its main goal is to use modeling tools to automatically or manually model the structure, spatial location, cultural and physical characteristics, and attribute information of features. Despite its strong dynamic 3D expressiveness, 3D animation lacks real-time interactivity. People are passive, so any changes to the plan or the observation route must be recalculated, and the results will not be visible for several days or even weeks. People can conduct an immersive and all-around review of the city or planning scheme in a dynamic and interactive manner in a close-to-real 3D environment in the 3D digital city [29]. People can view the scene from any angle, distance, or degree of precision; they can select and switch between a variety of sports modes; and they can control the browsing route.

With the deeper exploration of geospatial information and more extensive applications, the 3D model of single building or group of buildings has been unable to meet people's demand for global spatial information. The 3D model of building group has gradually become urban planning and management, architectural design, and important infrastructure in many fields such as emergency response, public safety, environmental protection, real estate and commercial site selection, and traffic navigation. In computer vision and computer graphics, the main research includes the modeling and display of 3D objects, that is, 3D modeling from the outside to the inside, and the modeling and display of earlydimensional real scenes, that is, the 3D modeling and display from the inside out.

Remote sensing image segmentation [30] is one of the key links in the subframework of building group target recognition. Aiming at the problems of existing remote sensing image segmentation methods that are difficult to comprehensively consider various features such as spectrum, shape, texture, lack of multiscale features, slow speed, low accuracy, and difficulty in adapting to the segmentation needs of urban building groups, an object-oriented multiscale is proposed. The region merging and segmentation method and the fast region merging and segmentation method based on quantified merging cost are both demonstrated to be feasible in terms of efficiency and accuracy through a series of experiments. In general, the human eye perceives the overall geometry and texture characteristics of the building model in high-resolution scenes, but it is not sensitive to the perception of these fine geometric components. Even in some cases, structures that are too fine and complex can cause people to recognize them. Knowing entails responsibilities. As a result, in the $3 \mathrm{D}$ architectural mesh model, the complex detailed structure and its fine parts are the objects with the highest priority for simplification. To better maintain the original architectural model, the unnecessary triangular meshes of the fine parts of these models can be merged or streamlined. While maintaining its visual and geometric features, the amount of model data is significantly reduced. The 3D spatial data model is shown in Figure 1.

The multisource $3 \mathrm{D}$ space model is transmitted to the client. If the data cannot be used directly, the model needs to be reconstructed. The role of reconstruction is to enable the system to access multiple data or to use the data structure defined by the developer to customize many special functions, so that it does not need to be restricted by the existing data structure. Developers can use their own defined data structure to realize the customization of special functions in the program, with a higher degree of freedom. The level of detail technology uses a set of entity level detail models with different levels of complexity to describe the same object and switches among these detail models according to the distance of the viewpoint or other standards in the graphics rendering, so as to change the complexity of the scene in real time. $3 \mathrm{D}$ information extraction and coordinate correction are another key link in the subframework of building group target recognition. On the basis of obtaining the height information, according to different accuracy levels, three types of building group layer number estimation models are summarized, which provide a variety of ways to quickly obtain the layer number information of a large number of buildings.

Texture mapping is a technique for creating a special effect or a sense of realism by mapping a two-dimensional image to a geometric shape, rather than a geometric model. Fine modeling and nonfine modeling are distinguished in the actual modeling process based on the various research foci. This section of the scene does not require fine modeling 


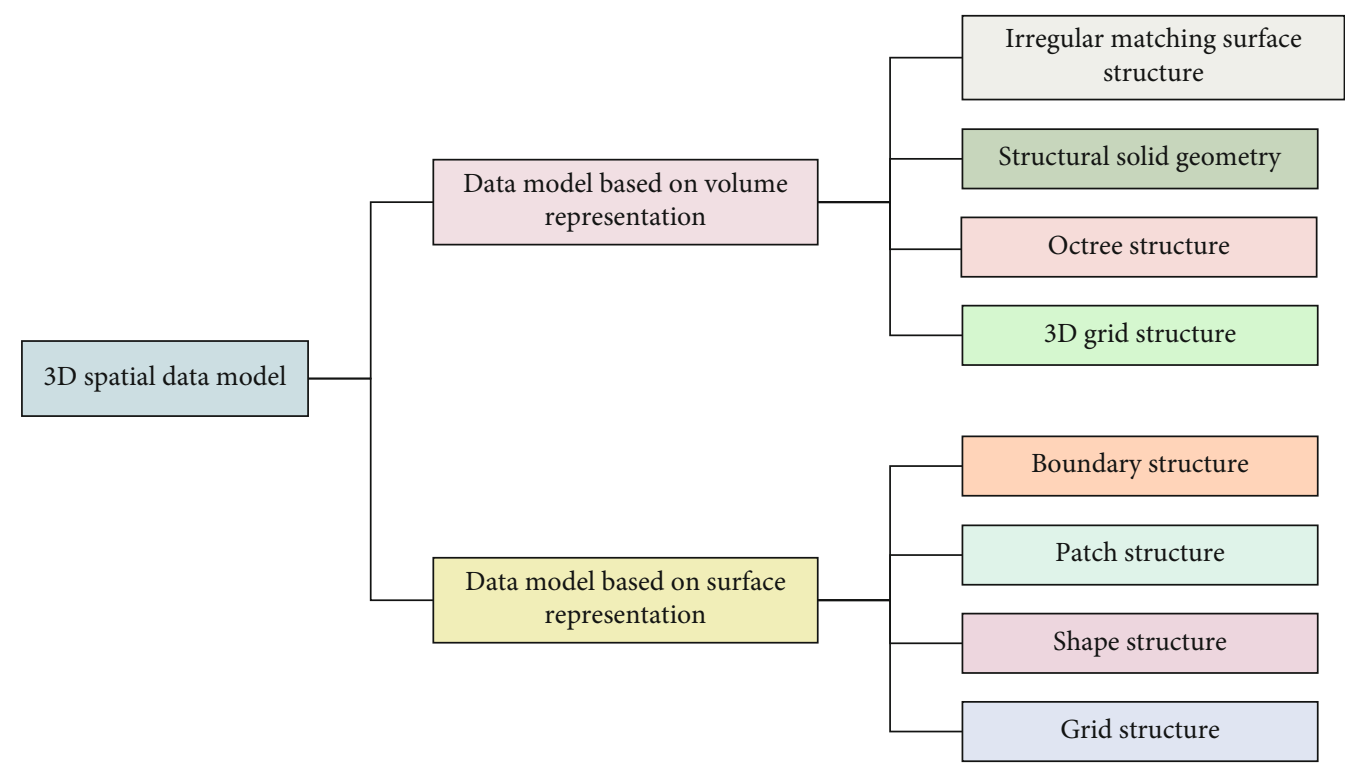

Figure 1: 3D spatial data model.

because there are so many irregular objects in it. Texture mapping technology can be used to simulate the creation of $3 \mathrm{D}$ models for this part of the ground objects. This takes into account the ground features' fidelity as well as the system's operating speed. People must figure out a way to solve this problem so that the original model's visual characteristics are preserved and the model's surface uses as few points as possible. By reducing the number of polygons with inconspicuous details on the model's surface, mesh simplification reduces the model's complexity while still meeting human visual requirements to the greatest extent possible.

3.2. 3D Modeling of Urban Buildings. The main goal of photogrammetry and remote sensing has always been to automatically reconstruct geometric models of $3 \mathrm{D}$ terrain surfaces and features from 2D images and even realistic models with photographic texture. The photogrammetry method allows for the automated acquisition of geometric and surface texture information for a large number of complex 3D city models at the same time. In comparison to ordinary $3 \mathrm{D}$ applications, the $3 \mathrm{D}$ building environment has a large data scale, a rich and diverse data type set, and a high level of scene graphic data complexity, all of which add to the data management and scene description challenges. The application is hampered by massive $3 \mathrm{D}$ spatial data with high complexity, and 3D spatial data compression and transmission have become the core technology of the virtual GIS environment. Faster network transmission can be achieved through 3D model data compression, allowing the real scene of a fine 3D model to be browsed online in real time and a virtual scene with a strong sense of reality to be realized.

For the description of buildings, the overall shape of the building, the projected outline of the building, and the local components of the building are generally explained from the following three aspects. The overall shape of a building refers to the overall appearance of a building, which can reflect the modeling complexity of a building. The projected outline of a building refers to the projected shape of the building on the ground, which can explain the top view of the building. More importantly, the coordinates of each point on the outline of the building can be used to accurately locate the building on the 3D terrain model. The local components of the building are used to describe the local building shapes of the building. Figure 2 is the decomposition of 3D model structure relationship of single building.

An important criterion in $3 \mathrm{D}$ reconstruction algorithm is system response time. The rapid response of the system can ensure the stability of the system. An important branch of the development of 3D graphics technology is the development of graphics hardware technology. In order to support and give full play to the advantages of graphics hardware technology, the system will provide optimal support for parallel processing functions provided by hardware. Different from traditional network services, 3DGIS network services are accompanied by massive spatial data and related attribute data, which include data types such as terrain, image, vector, model, attribute, texture, sound, text, and multimedia. With the development of network technology, efficiency has become a difficult problem in network service. In order to obtain suitable building object elements, it is necessary to divide the details of building elements into different levels. The building object elements can be obtained by dividing according to the combination of the internal structure of the building and the overall shape of the building. This hierarchical relationship of vertically lower level voxels is bound up and merged into higher level voxels, and the same kind of voxel components in the horizontal direction is combined voxel components in the same level through constraint combination tiling relationship. The $3 \mathrm{D}$ model of the building is expressed as a hierarchical constraint relationship model of vertical building voxel components and tiling constraint model of horizontal building voxel components. 


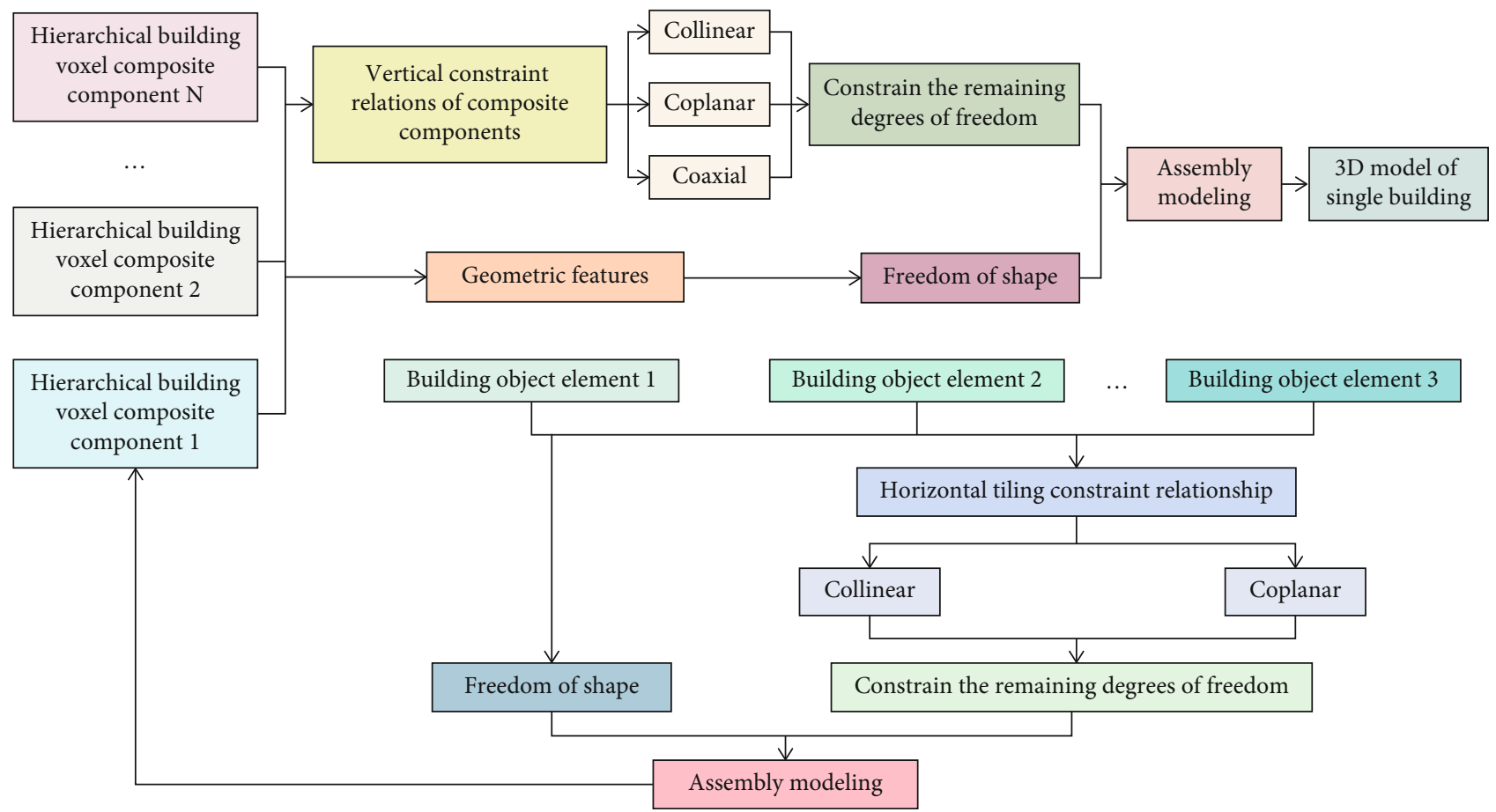

FIGURE 2: Structural relation decomposition of the single building 3D model.

The data organization module uses airborne laser scanning to collect geometric feature data from the urban surface. The data of newly developed areas is obtained based on the design results of urban planning designers for urban planning. Because polygonal patches can create 3D mesh models with a variety of topological structures, it is simple to express the $3 \mathrm{D}$ mesh model structure, and mesh models with various resolutions can be created by increasing or simplifying the polygonal patch operation. As a result, the majority of mesh models in use today are made up of planar polygons. The digital terrain model of the triangulation network is built using data from the terrain point cloud in the data organization module. The plane triangle is constructed using the control points of aerial laser scanning as vertices to approach the urban surface using the principles of nonintersection and nonrepetition. The triangulation digital ground model can accurately present the urban surface if the control points of aerial laser scanning are distributed reasonably and the control density is appropriate.

Select a segment of the original polygon, and set the straight line on one side of the ideal rectangle corresponding to this segment as $\Delta t p_{t} u_{t}(y)$. In order to simplify the calculation, the method in this paper assumes that the straight line is parallel to the corresponding side of the circumscribed rectangle of the minimum area, and the straight line is divided into $1+\Delta$ $t u_{t}\left(y^{\prime}\right)$. The formula for the sum of square errors between the segment and the straight line in the original polygon is

$$
\begin{gathered}
P_{t+\Delta t}=p_{t}+\Delta t p_{t} u_{t}(y) \\
P_{t+\Delta t}=p_{t}\left(1+\Delta t u_{t}(y)\right)+p_{t}\left(1+\Delta t u_{t}\left(y^{\prime}\right)\right) .
\end{gathered}
$$

$P_{t+\Delta t}$ finds the first-order partial derivative of $y$. When the first-order partial derivative is 0 , the error is the smallest, so

$$
\begin{gathered}
s_{t+\Delta t}(y)=\frac{p_{t+\Delta t}}{P_{t+\Delta t}}=\frac{p_{t}+\Delta t p_{t}(y)}{p_{t}\left(1+\Delta t u_{t}(y)\right)+p_{t}\left(1+\Delta t u_{t}\left(y^{\prime}\right)\right)}, \\
s_{t+\Delta t}(y)=\frac{p_{t+\Delta t}}{P_{t+\Delta t}}=\frac{s_{t}(y)\left(1+\Delta t u_{t}(y)\right)}{s_{t}(y)\left(1+\Delta t u_{t}(y)\right)+s_{t}\left(y^{\prime}\right)\left(1+\Delta t u_{t}\left(y^{\prime}\right)\right)} .
\end{gathered}
$$

The fitted straight line equation for this segment is

$$
s_{t+\Delta t}(y)-s_{t}(y)=s_{t}(y) \frac{\Delta t u_{t}(y)-\Delta t \bar{u}_{t}^{p}}{1+\Delta t u_{t}} .
$$

The fitting linear equations of the other three segments are obtained by the same method. Finally, get the intersection point of the fitting straight lines; that is, get the final optimization result of regular rectangle.

In a 3D architectural design system, the geographic affairs and landscape object information are complicated, and the data is vast. It is nearly impossible to achieve real-time visualization of massive data using conventional visualization technology under current hardware conditions. As a result, some unique scene data processing strategies must be implemented. The exclusive $3 \mathrm{D}$ model database and some auxiliary $2 \mathrm{D}$ basic geographic information data are established using Yijing map platform's 3D model import interface, ensuring that the 3D model accurately reflects the $3 \mathrm{D}$ information of terrain and features, 


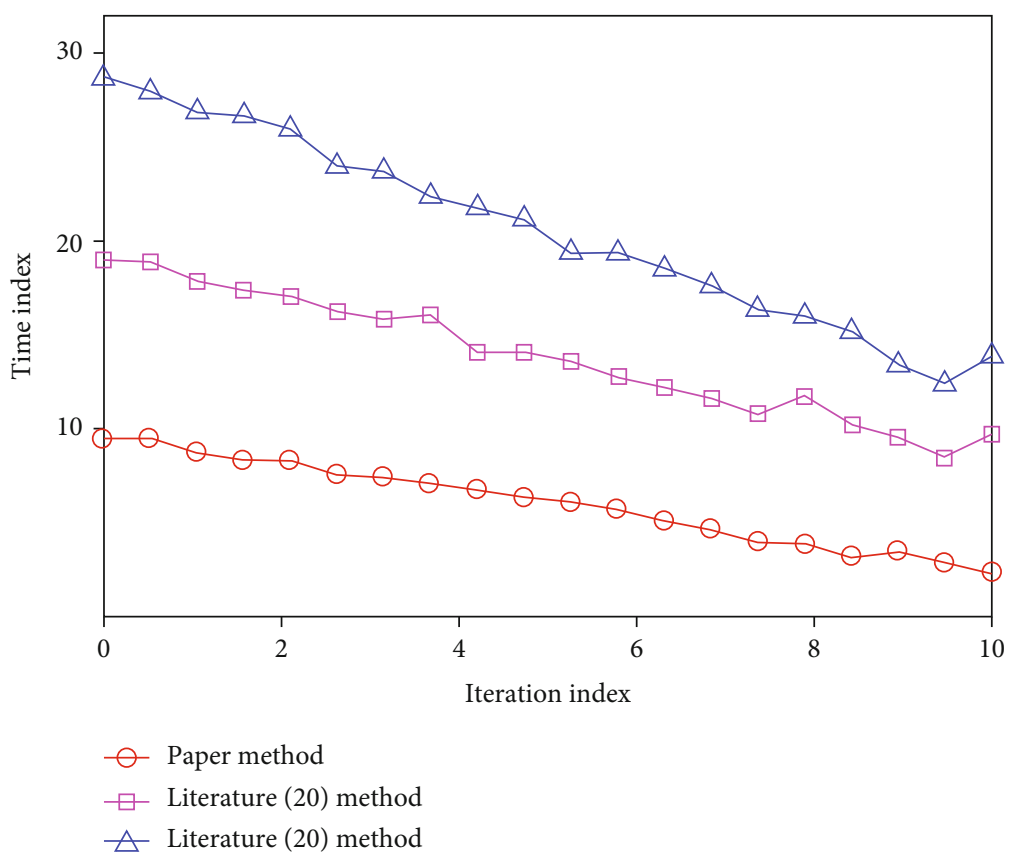

Figure 3: Time cost comparison result.

avoids $2 \mathrm{D}$ data confusion to the greatest extent possible, and facilitates later 3D model update and maintenance. The terrain 3D information table, ground $3 \mathrm{D}$ information table, and subordinate auxiliary association table are the main tables in the $3 \mathrm{D}$ model database.

When building reconstruction is carried out, for applications with different levels of detail, the voxel composition is determined according to professional requirements and production methods, and the details are gradually added or deleted according to the order from primary to secondary, so as to generate models with different levels of detail. For 3D modeling of urban buildings, it is necessary to determine a group of voxels describing the buildings. These voxels are assembled and combined to form the $3 \mathrm{D}$ model of the building.

Because each point's adjacent points form an edge and each edge can calculate a generation value, we use the smallest generation value of the edge formed by a vertex and all adjacent points as the generation value of the edge to be folded corresponding to that point in actual operation. Fold from the smallest value of the edge generation to be folded corresponding to the point in half-folding operation. When the distance between the viewpoint and the node is smaller during the rendering of terrain, this node indicates that the level of detail of the terrain is higher. Because each node can represent the size of a rectangular area, there may be differences, so if this is used as a standard, it may lead to unnecessary errors. The degree of detail in the model's feature area is reflected in the vertex curvature. Curvature is generally small in the flat area of a 3D model. The curvature is larger in the feature areas of the $3 \mathrm{D}$ model, such as creases and inflection points. Take any triangular patch $T_{0}$, and record its three vertices as $I_{t}, C_{t}$, and $Q_{t}$, respectively; then, the unit normal vector of triangular patch $T_{0}$ can be expressed as

$$
R_{t}\left(p_{1 t}, Q_{t}\right)=p_{1 t} \cdot \min \left(I_{t}+Q_{t}, D_{t}\right)-\left(p_{0 t} \cdot Q_{t}+C_{t} \cdot \mathrm{AI}_{t}\right)+R_{t-1}
$$

By weighting the area of the normal vector of the triangle surface in the first-order neighborhood of vertex $I_{t}$, the vertex normal vector of $C_{t}$ can be calculated $I_{t+1}$, and the vertex normal vector of vertex $I_{t}$ can be calculated as follows:

$$
I_{t+1}=I_{t}+Q_{t}-\min \left(I_{t}+Q_{t}, D_{t}\right)=\max \left(I_{t}+Q_{t}-D_{t}, 0\right) .
$$

After the normal vector of the vertex $I_{t}$ is obtained, the curvature $\mathrm{AI}_{t}$ of the vertex can be calculated, as shown in the following equation:

$$
\mathrm{AI}_{t}=\frac{\left(I_{t}+Q_{t}\right)}{2} \cdot \frac{\left(I_{t}+Q_{t}\right)}{D_{t}},
$$

where $I_{t}+Q_{t}$ is the angle between the normal vector of the vertex and the $k$-related triangle faces.

If the level of detail difference between adjacent nodes is 1 , when the viewpoint distance and the node distance are relatively close, the accuracy of the whole terrain can be improved by adding edges. When the distance between viewpoints and nodes is far apart, we should use the form of truncated edges, which can also reduce the level of detail of terrain and further reduce the number of meshes to be rendered, but it should not affect the visual effect. Choosing a suitable edge as a folding edge is the key of this half-edge folding algorithm. Good selection of folding edge is usually 


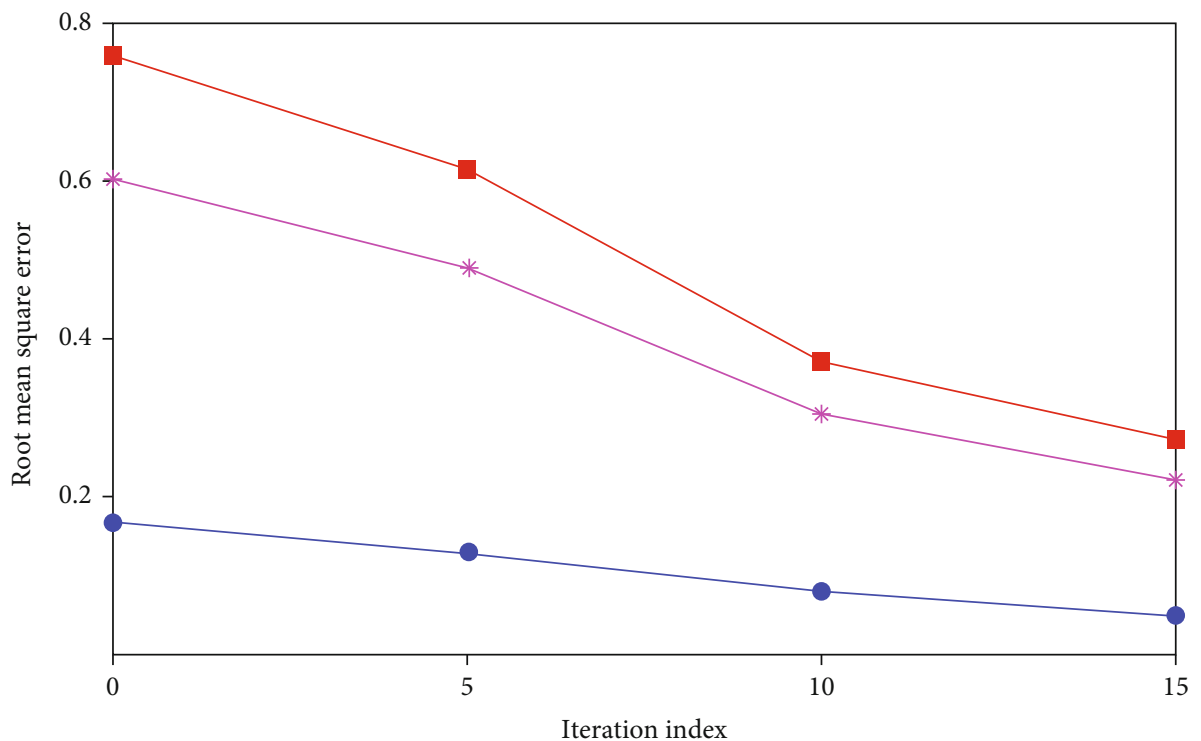

- Literature (21) method
$*$ Literature (20) method
- - Paper method

FIGURE 4: Root mean square error comparison result.

after half-folding, which makes the visual change of the model have the least influence.

The line number and column number of the frame are combined into a unique code, which is used as the frame number, according to the spatial range of data division. Data of various granularities are stored in the appropriate levels of the pyramid structure, according to the coding rules. It is also necessary to obtain the spatial range of the model's outer bounding box and construct the spatial association between the model and the modeling unit for spatial data such as $3 \mathrm{D}$ building models, in addition to dividing its geographic space according to spatial location information. Because there is no need for a grid with a high level of detail, the edge deletion method is used for rendering when the distance between the viewpoint and the node is greater than the set threshold.

\section{Result Analysis and Discussion}

Similar to popular 3D animation, powerful 3D modeling technology is also used to create realistic $3 \mathrm{D}$ scenes and "reproduce" planned projects. The virtual environment created by urban simulation technology, on the other hand, is a collection of digital models based on real data that strictly adhere to engineering project design standards and requirements and is part of the simulation system. To test the applicability of the method proposed in this paper in urban architectural design, a city was chosen as the research object, and the method was applied to the research object's urban architectural design, yielding the following application results. The immersiveness and interactivity of a $3 \mathrm{D}$ urban planning system can provide users with not only a strong and vivid sensory impact but also an immersive experience.

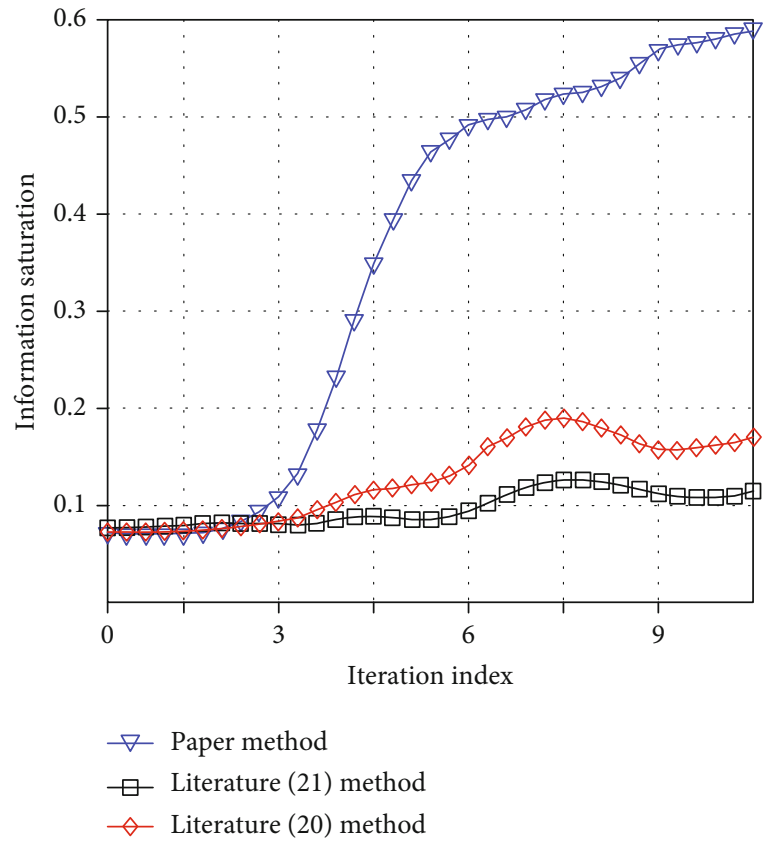

FIGURE 5: Information saturation comparison result.

It can also be used in conjunction with GIS data via its data interface to obtain project data in a real-time virtual environment, which is useful for the planning, design, bidding, approval, and management of large-scale complex engineering projects. In addition, a 3D urban planning system can be combined with information to enable $3 \mathrm{D}$ spatial data to be operated remotely.

Primitive classification roughly includes five steps: feature calculation, feature selection, classification, adjustment, 


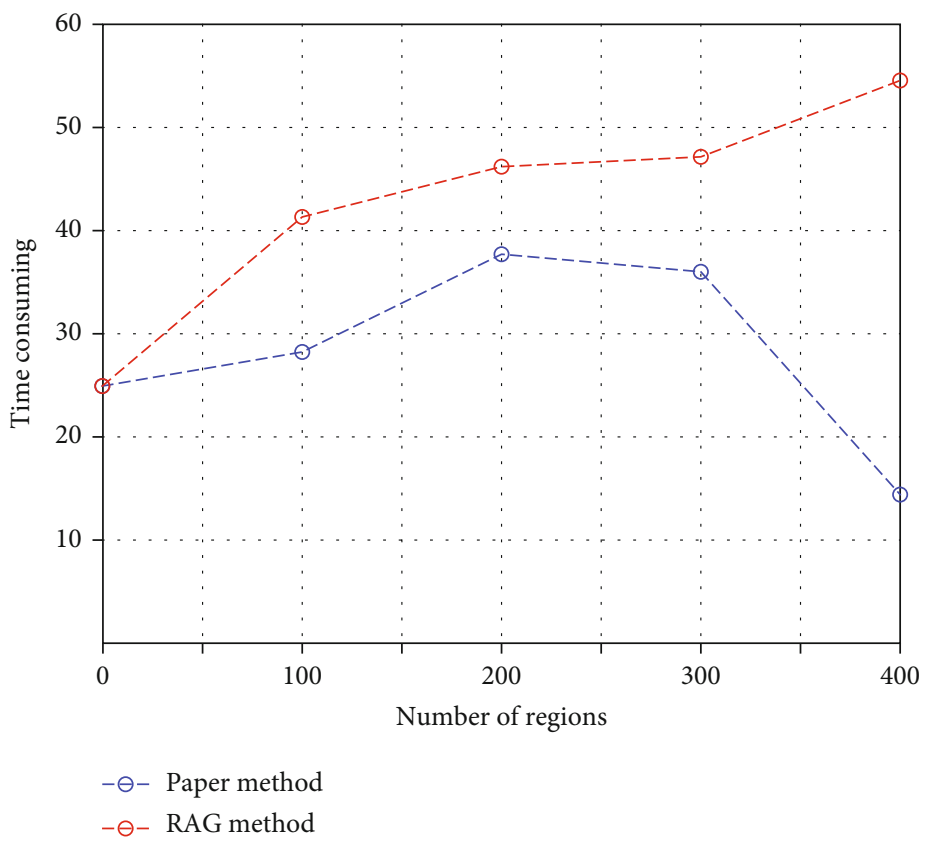

FIGURE 6: Relationship between regional quantity and merger time consumption.

and primitive combination. Feature calculation refers to the calculation of the spectrum, shape, texture, and other feature data for each primitive. In this experiment, the average brightness value, standard deviation, and ASM are calculated. Feature selection refers to selecting several features from all calculated features as the basis for classification, and all three features mentioned above are selected in this experiment. Classification refers to dividing primitives into multiple categories according to the selected feature numbers. In order to verify the effectiveness of the algorithm proposed in this paper, the method in this paper is compared with the method in the literature. The comparison result of time cost is shown in Figure 3. The comparison result of root mean square error is shown in Figure 4. The information saturation comparison result is shown in Figure 5.

Time cost can reflect the running speed of the system, and the smaller the value, the faster the running speed of the system. The normalized root mean square error description system describes the characteristics of the main buildings and landscapes in the city. The smaller the value, the more accurate the characteristics are. Information saturation reflects the visual presentation effect of system planning, and the larger the value, the better the visual presentation effect of system planning. From Figures 3-5, it can be seen that all parameters of this method are better than those of the comparison method, which shows that this method has remarkable performance advantages.

The small area formed after image segmentation is only a discrete grid pixel point set, and the points in the set are not related to each other except for the same or similar color values. Therefore, it is impossible to form an object with independent individual concept, and it is impossible to carry out object-oriented image analysis. Therefore, we use the vectorization function module in the system to vectorize the image segmentation map. In fact, the index is a set of vertex IDs corresponding to a triangle in triplet. Each folding will reduce the triangle, so we need to eliminate the reduced triangle from it. At the same time, due to the removal of a certain point, the ID number of the point in the point set needs to change accordingly. In order to keep the point indexed unchanged, the index number less than the ID number of the deleted point in the index should be reduced by 1 . In order to verify the advantages of the new method in terms of efficiency and accuracy, we made further comparative experiments and obtained the results as shown in Figures 6 and 7.

Figure 6 shows the relationship between the number of regions and the merging time. From Figure 6, it can be found that the merging speed advantage of the new method will become more and more obvious with the increase of the number of scenes in the initial segmentation region of the image. Figure 7 shows the relationship between cost spacing and relative segmentation accuracy (RSA). It can be seen from Figure 7 that RSA gradually improves with the reduction of cost distance. The accuracy can already meet most application requirements.

Due to the complexity of ground objects in remote sensing images, the phenomenon of different spectra of objects and different spectra of foreign objects frequently occurs, resulting in various classification errors in the initial classification results of elements, necessitating some manual adjustment. There are three types of adjustment methods: (1) correcting the local misclassification phenomenon inside and around the building roof, (2) correcting buildings with similar roof and facade colors, and (3) correcting other structures that are close to the color of the roof and are incorrectly divided and adjusting them to other types The cost of edges that correspond to points is recalculated when the topological structure of points is updated. The 


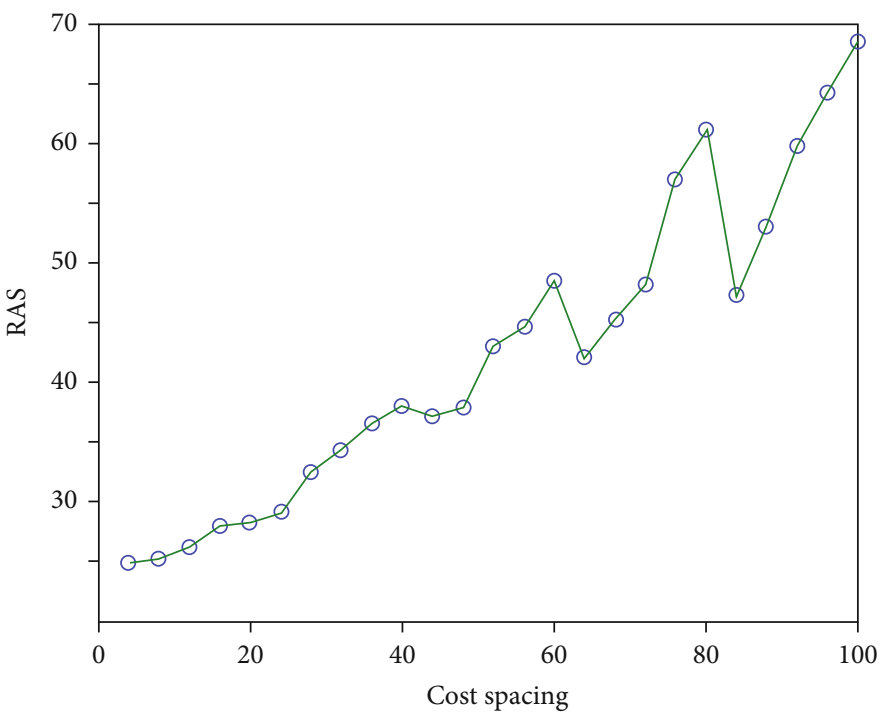

FIgURE 7: Relationship between cost interval and RSA.

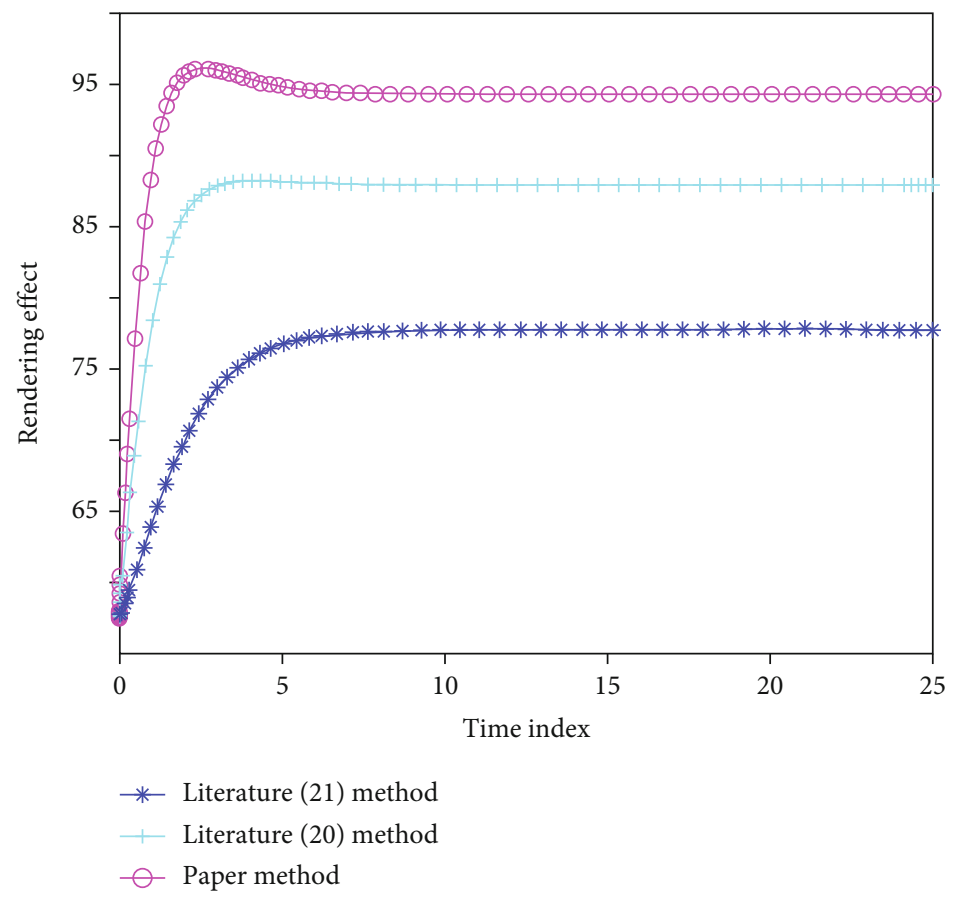

FIGURE 8: Different algorithm processing results.

topological relationships of the adjacent points of the vanishing point $U$ have changed after folding an edge. According to the law of edge folding operation, the topological relations of these points are reestablished at this point. The control group used the algorithm in the literature for $3 \mathrm{D}$ reconstruction and the LOD algorithm for rendering, while the terrain rendering algorithm based on improved LOD was used for $3 \mathrm{D}$ reconstruction of the urban landscape. Figure 8 shows the simulation results.

As shown in Figure 8, the effect of 3D reconstruction with literature algorithm and rendering with LOD algorithm is not good, but the processing result with the algorithm pro- posed in this paper is good, which proves the effectiveness of the algorithm proposed in this paper.

Determining the spatial range of subregions is an important part of data framing, and the spatial range of subregions affects the data organization, transmission, and rendering effect to a certain extent. The divided subarea is too small, which easily leads to frequent data access and reduces its transmission efficiency. The divided subarea is too large, and too many spatial data are retrieved at one time, which may affect the rendering effect of spatial data. According to the $3 \mathrm{D}$ spatial data division method of large-scale scene designed in this paper, the experimental area is divided into 
data. As long as the points disappeared after the folding operation are stored in the heap in turn, the details of the simplified model can be restored from the heap as needed after the simplification is completed.

The geometric simplification method of semifolded edges can delete triangles on the mesh surface without generating new vertices and can store the information of points, lines, and surfaces removed in the simplification process to construct geometric models with different resolutions. It makes up for the shortcomings of other simplified algorithms from different angles. At the same time, as the detailed information of each simplification is recorded, continuous transition can be directly carried out in meshes with different resolutions. From the rendering results in this chapter, it can be seen that the algorithm in this paper effectively reduces the amount of data transmitted by the network of large-scale urban scene models and reduces the rendering pressure of the client on the premise of retaining the important geometric features of the scene.

\section{Conclusions}

Digital earth gave birth to the concept of city, and digital city is an integral part of it. Furthermore, at this time, the digital city is an unavoidable consequence of globalization of information. As a result, urban architectural design must rely on the first important step of the digital city and use it as a major source of content in order to complete the project. When a building, as a small unit, is knocked down or rebuilt in the $3 \mathrm{D}$ model of the city studied in this paper, the state of the real world is altered. It is critical to use the evolution from different historical states to realistic states if we want to observe the development of a city. The geometric data and texture data of an urban 3D model are processed using current mature technology in this paper, and the geometric data is simplified using a half-folded mesh, which not only simplifies the model but also saves the simplified record, which can be used to restore the model's details. Compressing texture data with wavelet technology saves space and speeds up network transmission. In this paper, a 3D reconstruction algorithm based on Kalman filter correction and a terrain rendering algorithm based on LOD are proposed. Divide the $3 \mathrm{D}$ model on the data, and store the geometric data, texture data, and attribute data in tables. A set of three-dimensional management of urban 3D model data in space, time, and form, which can meet many different business needs, was formed. The simulation results show that the algorithm is effective in $3 \mathrm{D}$ reconstruction and rendering of urban landscape, and it is worth popularizing.

\section{Data Availability}

The data used to support the findings of this study are included within the article.

\section{Conflicts of Interest}

The authors do not have any possible conflicts of interest.

\section{Acknowledgments}

This work is funded by the characteristic innovation project of colleges and universities of Guangdong Provincial Department of Education. It is a phased achievement of "little Taishan, big world-Research on the promotion of architectural culture of Taishan Qiaowei," No. 2019wtscx018. And this work obtained funding from Guangdong University of Foreign Studies to introduce talents research startup project.

\section{References}

[1] A. Perisic, M. Lazic, and B. Perisic, "The Extensible Orchestration Framework approach to collaborative design in architectural, urban and construction engineering," Automation in Construction, vol. 71, no. 2, pp. 210-225, 2016.

[2] W. Kuai, "A case study on architectural and urban design of urban waterfront area based on green ecology," $C$ e Ca, vol. 42, no. 3, pp. 938-944, 2017.

[3] L. Li, "Application of renewable energy in architectural design and its reasonable utilization," Revista de la Facultad de Ingenieria, vol. 32, no. 14, pp. 766-770, 2017.

[4] F. Jiang, "Design and realization of $3 \mathrm{~d}$ simulation system for large-scale architectural landscape based on BIM technology," Revista de la Facultad de Ingenieria, vol. 32, no. 11, pp. 619625, 2017.

[5] A. Ambole, "Embedding design in transdisciplinary research: perspectives from urban Africa," Design Issues, vol. 36, no. 2, pp. 28-40, 2020.

[6] N. T. Dulworth, J. T. Strong, and C. Giegold, "Ray methods in architectural acoustic design," The Journal of the Acoustical Society of America, vol. 148, no. 4, pp. 2525-2525, 2020.

[7] B. Pirouz, M. Turco, and S. A. Palermo, "A novel idea for improving the efficiency of green walls in urban environment (an innovative design and technique)," Water, vol. 12, no. 12, p. $3524,2020$.

[8] L. Massetti, M. Petralli, G. Brandani et al., "Modelling the effect of urban design on thermal comfort and air quality: the SMARTUrban project," Building Simulation, vol. 12, no. 2, pp. 169-175, 2019.

[9] S. Otero, "Enhancing home recording spaces through architectural acoustics design," Journal of the Acoustical Society of America, vol. 141, no. 5, pp. 3994-3995, 2017.

[10] H. Kohei, "Smart city from a perspective of urban design," The Journal of The Institute of Electrical Engineers of Japan, vol. 139, no. 3, pp. 136-139, 2019.

[11] C. P. F. Shields, D. T. Rigterink, and D. J. Singer, "Investigating physical solutions in the architectural design of distributed ship service systems," Ocean Engineering, vol. 135, no. 5, pp. 236-245, 2017.

[12] R. Han, Y. Gao, and D. Shao, "Research on the application of building information model technology in the design of urban residential buildings in cold region," International Journal of Smart Home, vol. 10, no. 5, pp. 183-194, 2016.

[13] C. Sweetapple, M. Astaraie-Imani, and D. Butler, "Design and operation of urban wastewater systems considering reliability, 
risk and resilience," Water Research, vol. 147, no. 12, pp. 1-12, 2018.

[14] D. Halsted, "The origins of the architectural metaphor in computing: design and technology at IBM, 1957-1964," IEEE Annals of the History of Computing, vol. 40, no. 1, pp. 61-70, 2018.

[15] M. Kendin, A. Nikiforov, R. Svetogorov, P. Degtyarenko, and D. Tsymbarenko, "A 3D-coordination polymer assembled from copper propionate paddlewheels and potassium propionate $1 \mathrm{D}$-polymeric rods possessing a temperature-driven single-crystal-to-single-crystal phase transition," Crystal Growth and Design, vol. 21, no. 11, pp. 6183-6194, 2021.

[16] J. Diao, C. Xu, A. Jia, and Y. Liu, "Virtual reality and simulation technology application in 3D urban landscape environment design," Boletin Tecnico/Technical Bulletin, vol. 55, no. 4, pp. 72-79, 2017.

[17] Y. N. Tang and J. S. Liu, "Architectural design of urban traffic hub based on regionalism and culture," Journal of Railway Engineering Society, vol. 34, no. 10, pp. 78-82, 2017.

[18] M. Khalil, "Idea generating techniques in architectural design education: exploring students' perceptions," International Journal of Engineering Education, vol. 37, no. 1, pp. 65-85, 2021.

[19] M. Yao and Y. Wu, "Study on architectural space design of modern urban landscape," Revista de la Facultad de Ingenieria, vol. 32, no. 14, pp. 878-882, 2017.

[20] A. Agirbas and E. Ardaman, "Macro-scale designs through topological deformations in the built environment," International Journal of Architectural Computing, vol. 15, no. 2, pp. 134-147, 2017.

[21] D. S. Woolworth, "Implications of modern classroom architectural design and pedagogy on meeting ANSI S12.60 national classroom acoustics standard requirements," The Journal of the Acoustical Society of America, vol. 148, no. 4, pp. 26292629, 2020.

[22] A. Groenewolt, T. Schwinn, L. Nguyen, and A. Menges, “An interactive agent-based framework for materializationinformed architectural design," Swarm Intelligence, vol. 12, no. 2, pp. 155-186, 2018.

[23] H. Salehi and E. Akbari, "Design of architectural models to enhance the security of buildings and urban areas against military with passive defense approach," Acta Technica CSAV (Ceskoslovensk Akademie Ved), vol. 63, no. 5, pp. 683-700, 2018.

[24] N. Wu and M. Wang, "The integration of procedural information in traditional architectural design," Computer-Aided Design and Applications, vol. 16, no. 1, pp. 13-24, 2018.

[25] Y. Miao, R. Koenig, K. Knecht, K. Konieva, P. Buš, and M. C. Chang, "Computational urban design prototyping: interactive planning synthesis methods-a case study in Cape Town," International Journal of Architectural Computing, vol. 16, no. 3, pp. 212-226, 2018.

[26] M. Tamke, P. Nicholas, and M. Zwierzycki, "Machine learning for architectural design: practices and infrastructure," International Journal of Architectural Computing, vol. 16, no. 2, pp. 123-143, 2018.

[27] M. Dabaieh, M. Lashin, and A. Elbably, "Going green in architectural education: an urban living lab experiment for a graduation green design studio in Saint Catherine, Egypt," Solar Energy, vol. 144, no. 3, pp. 356-366, 2017.
[28] A. Jutraz and J. L. Moine, "Breaking out: new freedoms in urban (re) design work by adding immersive environments," International Journal of Architectural Computing, vol. 14, no. 2, pp. 103-118, 2016.

[29] X. Zhang, "Application and implementation of 3D printing technology in urban stereoscopic sculpture design," Boletin Tecnico/Technical Bulletin, vol. 55, no. 14, pp. 9-16, 2017.

[30] Y. Ding, X. Zhao, Z. Zhang, W. Cai, and N. Yang, "Graph sample and aggregate-attention network for hyperspectral image classification," IEEE Geoscience and Remote Sensing Letters, vol. 19, pp. 1-5, 2022. 